\title{
Uber das Verhalten des Blutplättchens bei einigen chirurgischen Erkrankungen, mit besonderer Berücksichtigung der Nierentuberkulose.
}

\author{
Von \\ Yasukatu Yabe. ${ }^{\dagger}$ \\ (矢部 保 岤) \\ (Aus der Chirurgischen Klinik der Universität, Sendai. \\ Direktor: Prof. Dr. Sh. Sugimura.)
}

Über das Verhalten des Blutplättchens bei chirurgischen Erkrankungen findet sich bisher nur wenig Literatur vor. Die bisherigen einschlägigen Forschungen, die besonders von seiten der Internisten vorgenommen wurden, betrafen hauptsächlich die Blut- und Krebskrankheiten und zwar die Magenkrebse. Hinsichtlich der chirurgischen Erkrankungen handelte es sich meistenteils um Bluterkrankheit und maligne Geschwülste, so dass ich hier nur die von $\mathrm{H} \mathrm{ueck} \mathrm{nennen}$ möchte. Auch sind wegen technischer Schwierigkeiten bisher verschiedene Methoden der Blutplättchenzählung angegeben, so dass dabei die Wahl der Methode in Frage kam. Dies veranlasste mich deshalb, Untersuchungen über das Blutplättchen bei einigen chirurgischen Erkrankungen, nach einer Nachprüfung der technischen Einzelheiten bei den bisherigen Methoden der Blutplättchenzählung, anzustellen. Diese meine Ergebnisse werde ich an Hand von 230 Untersuchungen mit der Lampertschen Methode bei einigen chirurgischen Erkrankungen und zwar bei Nierentuberkulosen im folgenden kurz berichten.

\section{Technische Vorbémerkungen.}

Fs sind bisher verschiedene Methoden der Blutplättchenzählung angewandt, unter denen sich nach verschiedenen Forschern die Methode von Foni o (zitiert bei $\mathrm{Na}$ ageli) als relativ am exaktesten erwies. Diese indirekte Methode setzt dagegen bei der Ausführung besondere technische Einübungen voraus. Die direkte Methode der Blutplättchenzählung von La mpert, die 1931 veröffentlich wurde, ist erst von wenigen Autoren nachgeprüft worden. Ich habe mich bei meiner Blutplättchenzählung und zwar bei Gesunden der beiden 
genannten Methoden gleichzeitig bedient und die Ergebnisse dann miteinander verglichen. Das Resultat war, dass die Methode von Lampert, auf die ich weiter unten noch zurückkomme, ein konstantes Frgebnis lieferte und deshalb zuverlässiger zu sein scheint. Bei der weiteren Blutplättchenzählung bei Gesunden sowie bei einigen chirurgischen Erkrankungen wurde also die Lampertsche Methode verwendet. Alle diese meine Untersuchungen geschahen als Regel morgens, nüchtern, in der Bettruhe und wurden nur ausnahmsweise und zwar bei dringender Notoperation direkt vor der Operation ausgeführt.

Utber die technischen Einzelheiten der genannten beiden Methoden der Blutplättchenzählung und zwar der L a mpertschen möchte ich auf die Originalarbeiten verweisen. Bemerkt sei nur noch, dass ich bei der Untersuchung mit der La m pertschen Methode die abgekühlte Tyrode sche Lösung verwendet habe, die bisher kaum von Forschern ausser Preis s beachtet wurde. Der Autor bediente sich dabei einer mit Heparin zugesetzten, abgekühlten Ty rod eschen Lösung und ich selbst konnte aber ohne Zusatz des Heparins, d. h. durch den Gebrauch einer frischen, nicht mehr als 1 Woche veralteten, nur einfach abgekühlten Ty rod e schen Lösung denselben Zweck erreichen. Meine besonders angestellten vergleichenden Untersuchungen beim Gebrauch der abgekühlten $\mathrm{T}$ y rod e schen Lösung mit oder ohne Zusatz des Heparins bestätigten die Richtigkeit dieser meiner Annahme wieder. Nebenbei sei auch bemerkt, dass ich bei der Blutplättchenzählung gleichzeitig das ganze Blutbild d.h. die Zahl der Erythrozyten und Leukozyten, den Prozentgehalt der Leukozytenarten, Hämoglobingehalt des Bluts sowie die Senkungsgeschwindigkeit der Erythrozyten berücksichtigt habe. Ferner wurde bei den Frauen die Menstruation, Schwangerschaft sowie Wochenbett und Laktation bei der Untersuchung ausgeschlossen.

\section{Ergebnisse meiner Untersuchungen.}

\section{Ergebnisse der Vorprüfung.}

Zuerst habe ich, wie schon erwähnt, die oben genannten zwei Methoden der Blutplättchenzählung bei 21 Gesunden beiderlei Geschlechts gleichzeitig angestellt und die Resultate miteinander verglichen. Es handelte sich dabei um 9 Männer vom 18. bis 55. Lebensjahre und um 12 Frauen vom 12. bis 26. Lebensjahre. Daraus ergab sich, dass die Blutplättchenzahl nach der Methode von Lampert bei gesunden Männern durchschnittlich 323,300 in $1 \mathrm{cmm}$ Blut mit den Grenzwerten von 275,000 und 335,000 , bei Frauen 354,200 in $1 \mathrm{cmm}$ Blut mit den Grenzwerten von 220,000 und 500,000 betrug, während die gleichzeitig nach der Fonioschen Methode erhobene Zahl durchschnittlich bei Männern 237,800 in $1 \mathrm{cmm}$ Blut den Grenzwerten von 103,000 und 408,000 , bei Frauen 257,400 in $1 \mathrm{cmm}$ Blut mit den Grenzwerten von 
100,000 und 428,000 ergab. Das zeigt einwandfrei, dass die La m pertsche Methode im Vergleich mit der von F on io relativ höhere Ziffern mit weit geringeren Schwankungen aufweist und somit ein konstanteres Resultat, auch bei Ungeübten, liefert. Auch die Autoren wie Perl und Behr, die die Methoden bei Gesunden und Kranken vergleichend untersuchten, erkannten schon die praktische Brauchbarkeit der La mpertschen Methode an. Auf Grund der Vorprüfung verwendete ich bei meinen weiteren Untersuchungen der Blutplättchenzahl bei Gesunden sowie bei chirurgischen Erkrankungen ausschliesslich die Lampertsche Methode.

\section{Ergebnisse bei Gesunden.}

Meine weiteren Untersuchungen über Blutplättchenzahl bei Gesunden erstreckte sich auf 51 Personen, d.h. 30 Männer vom 18. bis 50. Lebensjahre und 21 Frauen vom 16. bis 45. Lebensjahre. Ausserdem wurden auch einige kleine Kinder sowie Personen vorgerückten Alters untersucht.

Aus den Ergebnissen der Blutplättchenzählung bei meinen 51 Gesunden geht hervor, dass die Blutplättchen bei meinen Gesunden durchschnittlich 300,000 in $1 \mathrm{cmm}$ Blut betragen. Ihr Mittelwert beläuft sich aber bei Männern auf 304,000 mit den Grenzwerten von 255,000 und 360,000, bei Frauen auf 293,000 mit den Grenzwerten von 250,000 und 350,000. Wenn ich diese Ziffern mit denen der bisherigen japanischen Forscher vergleiche, so finde ich, dass meine Ziffern mit den von $\mathrm{Nakae}$ und $\mathrm{Nak}$ a mura (zitiert bei Oka moto) als physiologisch angegebenen Zahlen übereinstimmen. Auch geben die neueren Forscher, wie Behr, Perl, Dameshek und Preiss die Zahl um 300,000 als den physiologischen Wert an. Ferner zeigte die Blutplättchenzahl bei meinen 6 gesunden Kindern (Fall 52-57) verschiedenen Lebensjahres durchschnittlich einen weit höheren Wert als bei meinen gesunden Erwachsenen. Dies stimmt beinahe mit der Angabe von Keilmann (zitiert bei Naegeli) überein, dass die Blutplättchen bei Säuglingen und Kindern vermehrt und starken Schwankungen unterworfen sind. Weiter stellte ich bei meinen 4 Gesunden vorgerückten Alters, d. h. über das 60. Lebensjahr weit herabgesetzte Werte, d. h. in der untersten normalen Grenze liegende Werte fest. Auch fanden die Autoren wie Denner (zitiert bei Naegeli) und Okamoto eine verminderte Blutplättchenzahl im Greisenalter.

Hier sei kurz bemerkt, dass das bei der obigen Untersuchung von 
mir gewonnene Hämogramm der erwachsenen Gesunden einen niedrigeren neutrophilen Leukozyten- und einen dementsprechend höheren Lymphozytenprozentsatz im Vergleich zu dem Schillingschen, für die Europäer normalen Hämogramm aufweist, wie schon von Kitasima mitgeteilt. Bokelmann u. Nassan führten die nach dem Weltkrieg bei Soldaten und Zivilpersonen beobachtete Lymphozytose der gesunden Menschen auf die kohlehydratreiche und fett- und eiweissarme Ernährung zurück. Aus dem Ergebnis meiner vergleichenden Untersuchung der Blutplättchenzahl mit dem Blutbilde konnte ich bemerken, dass die Blutplättchenzahl, wie auch aus meinen Untersuchungen bei chirurgischen Erkrankungen hervorgeht, in inniger Beziehung mit der neutrophilen Leukozytose steht, worauf ich unten noch zurückkomme. Dasselbe gilt gewissermassen auch für die Senkungsgeschwindigkeit der roten Blutkörperchen.

III. Ergebnisse bei akuten Infektionen.

Ich stellte die Blutplättchenzählung bei 63 alkuten Infektionen an, worunter sich 51 von akuter Wurmfortșatzentzündung mit oder ohne ihre Komplikationen und 12 anderweitige Eiterungen der Weichteile fanden. Die 51 Appendizitisfälle wiesen ihre verschiedenen Stadien auf oder waren mit den appendizitischen Abszessen sowiePerforationsperitonitis kompliziert. Bei 8 Fällen von Appendicitis acuta simplex, die zur Frühoperation kamen, wurde das Blutplättchen im Blute in der Hälfte der Fälle vermehrt gefunden. Dabei betrug die Blutplättchenzahl durchschnittlich 360,000 in $1 \mathrm{cmm}$ Blut mit den Grenzwerten von 245,000 und 460,000 in $1 \mathrm{cmm}$ Blut und diese Vermehrung ging Hand in Hand mit der Neutrophilie im Blute einher. Unter den weiteren 5 intermediär operierten Fällen von Appendicitis acuta wiesen 2 eine Vermehrung der Blutplättchenzahl auf, so dass die Blutplättchenzahl dabei durchschnittlich 333,000 in $1 \mathrm{cmm}$ Blute mit den Grenzwerten von 270,000 und 340,000 in $1 \mathrm{cmm}$ Blut betrug. Bei 5 unter 14 Fällen von Appendicitis gangraenosa war die Blutplättchenzahl deutlich vermehrt und bei 8 unverändert, bei einem (Fall 80) ja sogar vermindert. Die durchschnittliche Blutplättchenzahl betrug dabei 357,000 in 1 cmm Blut mit den Grenzwerten von 248,000 und 580,000 in $1 \mathrm{cmm}$ Blut und zeigte auch einen Parallelismus mit der Anzahl der Neutrophilen im Blute. Bei dem Fall 80 wurde gleichzeitig mit der deutlichen Verminderung des Blutplättchens eine hochgradige Linksverschiebung der neutrophilen Leukozyten mit wenig ausgeprägter Neu- 
trophilie festgestellt. Nebenbei sei erwähnt, dass bei der Mehrzahl von Appendicitis gangraenosa die Retikuloxyten im Blute weit vermehrt gefunden wurden. Bei 7 Fällen des appendizitischen Abszesses betrug die Blutplättchenzahl durchschnittlich 350,000 in $1 \mathrm{cmm}$ Blut mit den Grenzwerten von 270,000 und 430,000 in $1 \mathrm{cmm}$ Blut, die Vermehrung des Blutplättchens war also dabei weniger ausgeprägt. Unter 5 Fällen der akuten diffusen Peritonitis appendikulären Ursprungs war die Blutplättchenzahl bei 2 (Fall 96 u. 99) stark vermehrt, bei einem Fall (Fall 97) dagegen stark vermindert, so dass ihr Mittelwert sich etwas erhöhte. Er betrug nämlich durchschnittlich 377,000 in $1 \mathrm{cmm}$ Blut mit den Grenzwerten von 225,000 und 545,000 in $1 \mathrm{cmm}$ Blut. Bei 12, im Intervall operierten Appendizitisfällen blieb eine Vermehrung oder Verminderung der Blutplättchenzahl ganz aus, so dass ihr Mittelwert 312,000 in $1 \mathrm{cmm}$ Blut mit den Grenzwerten von 265,000 und 355,000 in $1 \mathrm{cmm}$ Blut betrug. Dabei war auch keine Neutrophilie im Blut festzustellen.

Ferner wurde das Blutplättchen bei meinen 7 akuten Eiterungen, die Karbunkel, Mundbodenphlegmone, akute Myositiden, Periproktalabszess und Pyothorax umfasssten, in der Mehrzahl der Fälle deutlich vermehrt und oft gleichzeitig zusammen mit ausgeprägter Neutrophilie und nicht starker Linksverschiebung der Neutrophilen gefunden. Bei 2 Ausnahmefällen (Fall 121 u. 122) mit stark ausgeprägter Linksverschiebung der Neutrophilen war das Blutplättchen sogar stets vermindert.

Aus dem Obigen zeigt sich, dass bei meinen akuten Infektionen das Blutplättchen im allgemeinen bei ausgeprägter Neutrophilie und nicht stärkerer Linksverschiebung der Neutrophilen vermehrt gefunden wurde. Diese Vermehrung des Blutplättchens erschien dagegen bei wenig ausgeprägter Neutrophilie und deutlicher Linksverschiebung der Neutrophilen nur sehr wenig ausgeprägt. Daraus geht hervor, dass sich die Blutplättchenzahl je nach der Schwere der akuten Infektion und der dabei sich abspielenden Reaktion 'des Gesamtorganismus verschieden verhält, und bei schwerer Infektion mit herabgesetzter Reaktion des ganzen Körpers stets eine Verminderung des Blutplättchens zusammen mit den anderweitigen entsprechenden Erscheinungen wie der gehemmten Neutrophilie und der Linksverschiebung der Neutrophilen in Erscheinung tritt.

Anhangsweise sei hier erwähnt, dass die Blutplättchenzahl bei meinen 12 Fällen der chronischen d. h. ausserrenalen tuberkulösen Infektionen, nämlich bei Tuberkulose der Lymphdrüsen, der Pleura sowie 
der Knochen und Gelenke, nur innerhalb der normalen Grenzen bleib. Dabei war die Lymphozytose im allgemeinen kaum ausgeprägt und die Senkungsgeschwindigkeit der roten Blutkörperchen meistenteils nur in geringem Grade beschleunigt.

IV. Ergebnisse bei malignen Geschwülstèn.

Die Blutplättchenzahl untersuchte ich im ganzen bei 39 Fällen von malignen Geschwülsten, worunter sich 35 Krebse und 4 Sarkome fanden. Unter den 35 Krebsen handelte es sich bei 24 um Magenkrebse, von denen 13 mit Resektion, 11 mit Gastroenterostomie behandelt wurden. Das Blutplättchen wies bei den maligen Geschwülsten in allgemeinen eine mehr oder weniger deutliche Verminderung auf und seine Anzahl betrug bei Magenkrebsen durchschnittlich 268,000 in $1 \mathrm{cmm}$ Blut mit den Grenzwerten von 205,000 und 360,000 in $1 \mathrm{cmm}$ Blut. Diese Verminderung der Blutplättchenzahl war darunter bei den mit Resektion behandelten d. h. wenig fortgeschrittenen Fällen aber nicht ausgeprägt. Das Blutplättchen betrug dabei durchschnitt: lich 278,000 in $1 \mathrm{cmm}$ Blut (Grenzwerte : 220,000 und 360,000). Bei den weit fortgeschrittenen, mit Anastomose behandelten Fällen kam diese Verminderung noch deutlicher als bei den resezierten Fällen zu Tage, so dass das Blutplättchen dabei durchschnittlich 259,000 in 1 cmm Blut (Grenzwerte: 205,000 und 335,000) aufwies. Bei einigen Ausnahmefällen des Magenkrebses mit Lebermetastase (Fall 151, 152 u. 154) und mit zunehmender Aszites (Fall 153 u. 154) erschien die Verminderung des Blutplättchens noch ausgeprägter und das Blutplättchen belief sich bei Fall 53 auf 245,000 und bei Fall 151 auf 235,000 in $1 \mathrm{cmm}$ Blut. Demnach lässt sich wohl mit Recht sagen, dass das Blutplättchen bei Magenkrebsen mit dem Fortschreiten des lokalen Krankheitsprozesses und mit der zunehmenden Kachexie eine mehr oder weniger starke Neigung zur Verminderung, im Gegensatz zu der Angabe ron Rosenbaum, Helber und Hueck, aufweist.

Bei meinen weiteren 11 Fällen des Krebses, die den Krebs des Rektums, Oberkiefers und der Haut, vor allem auch Brustdrüsenkrebse enthielten, zeigte das Blutplättchen ebenfalls eine mehr oder weniger deutliche Verminderung erst beim Fortschreiten des lokalen Prozesses und bei der Metastasenbildung. Sonst blieb die Blutplättchenzahl dabei oft innerhalb der normalen Grenzen. Ferner wurde bei meinen Krebsfällen bemerkt, dass die Verminderung des Blutplättchens im grossen und ganzen mit der Linksverschiebung der Neutrophilen bei 
fehlender Leukozytose, wenn auch nicht so deutlich wie bei akuten Infektionen, doch in nicht geringem Grade Hand in Hand ging.

Ich konnte das Blutplättchen weiter bei 4 Sarkomen der Weichteile untersuchen. Dabei lag die Blutplättchenzahl innerhalb normaler Grenzen und das Blutbild zeigte auch keine Abnormitäten. Rosenbaum bemerkte bei Sarkomen eine Vermehrung, Gundermann und Helber dagegen eine Verminderung des Blutplättchens. Weiter habe ich das Blutplättchen bei 6 Fällen von akutem Tleus, 6 von Ulcus ventriculi et duodeni, 8 von Kröpfen und 3 von spontaner Gangrän untersucht. Bei Meusfällen wies das Blutplättchen, wie bei akuten Infektionen, eine Vermehrung bei mässiger Leukozytose und minimaler Linksverschiebung der Neutrophilen und eine Verminderung bei ausbleibender Leukozytose und starker Linksverschiebung der Neutrophilen im Blute auf. Bei Magen- und Duodenalulcus konnte ich im Gegenesatz zu Magenkrebs keine Verminderung des Blutplättchens feststellen. Bei 2 Basedowkröpfen (Fall 192 u. 193) war das Blutplättchen mässig deutlich vermindert. Bei sonstigen Kropftällen und bei spontaner Gangrän des Fusses wies das Blutplättchen einen normalen Wert auf.

Ausserdem konnte ich bei 6 Fällen von akuter Wurmfortsatzentzündung und bei 7 Fällen von Magenkrebs über den Einfluss der operativen Entfernung des Krankheitsherdes auf die Blutplättchenzahl im Blute serienweise Untersuchungen anstellen. Bei allen diesen Fällen kam die einmal vor der Operation vermehrt oder vermindert gefundene, bei Magenkrebsen meistenteils deutlich herabgesetzte Blutplättchenzahl nach erfolgter Operation mit der Zeit zur Norm zurück. Besonders bei 2 Füllen von Magenkrebs (Fall 153 u. 158) vermehrte sich das Blutplättchen 1 oder 2 Tage nach der Magenresektion mehr oder weniger deutlich und überschritt die normale Grenze, um dann in den darauffolgenden Tagen allmählich zum normalen Wert zurückzukehren. Diese vorübergehende Vermehrung des Blutplättchens direkt nach der Operation ging dabei mit der Vermehrung der roten und weissen Blutkörperchen im Blute sowie mit dem Hämoglobingehalt des Blutes Hand in Hand, was m. E. zum Teil auf die Eindickung des Blutes infolge der Operation zurückgeführt werden dürfte.

V. Ergebnisse bei chirurgischen

Nierenerkrankungen.

Ich untersuchte weiter die Blutplättchenzahl bei 74 Fällen der 
chirurgischen Nierenerkrankungen, worunter sich 45 von Nierentuberkulose, 9 von Nierensteinkrankheit, je 2 von Nierengeschwïlste und Cystenniere, 9 von sog. chronischer hämaturischer Nephritis, 4 von Pyelitis non-tuberculosa und 3 von Paranephritis acuta fanden.

Unter meinen 45 Nierentuberkulosen kamen 30 zur Nephrektomie wegen einseitiger Erkrankung. Es handelte sich dabei um 2 von Tuberkulose der Papillenspitze, 22 von käsig-kavernöser Nierentuberkulose, je 1 Fall der tuberkulösen Pyonephrosis und der Kittuiere und 4 von grobknotiger Form. Ausserdem fanden sich noch 5 einseitige, aber nicht operierte und 10 beiderseitige, inoperable Nierentuberkulosen. Bei sämtlichen Fällen von Nierentuberkulose war das Blutplättchen im allgemeinen mehr oder weniger vermindert und betrug durchschnittlich 257,000 in $1 \mathrm{cmm}$ Blut mit den Grenzwerten von 120,000 und 410,000. Bei der Tuberkulose der Papillenspitze blieb die Blutplättchenzahl'ganz normal mit dem ebenso normalen Blutbild. Bei den käsig-kavernösen Nierentuberkulosen verhielt sich die Blutplättchenzahl durchschnittlich etwas niedriger als bei Gesunden. Besonders war es bei den Fortgeschrittenen der Fall. Bei der leichten käsig-kavernösen Nierentuberkulose betrug nämlich das Blutplätṫchen durchschnittlich 292,000 in $1 \mathrm{cmm}$ Blut mit den Grenzwerten von 255,000 und 330,000 , bei der mittelschweren 273,000 in $1 \mathrm{cmm}$ Blut mit den Grenzwerten von 210,000 und 400,000 und bei dem Endstadium 273,000 in $1 \mathrm{cmm}$ Blut mit den Grenzwerten von 190,000 und 365,000. Eine Vermehrung des Blutplättchens wurde dabei niemals beobachtet. Das Blutbild zeigte dabei nur selten und besonders bei Endstadium eine leichte Linksverschiebung der Neutrophilen. Bei der tuberkulösen Pyonephrosis wurde das Blutplättchen nur etwas vermehrt, bei der Kittniere und der grobknotigen Form trotz normalem Blutbilde stark vermindert gefunden. Es muss aber bemerkt werden, dass bei diesen einseitigen Nierentuberkulosen oft auch gleichzeitig eine tuberkulöse Komplikation von seiten der Lungen, der Pleura oder des Darmes vorlag und auch diese Komplikation einen nicht zu unterschätzenden Einfluss auf die Blutplättchenzahl ausübte.

Ferner habe ich bei 15 einseitigen Nierentuberkulosen die Blutplättchenzahl vor und nach der Nephrektomie vergleichend untersucht. Dabei betrug das Blutplättchen durchschnittlich vor der Operation 262,700 in $1 \mathrm{cmm}$ Blut (Grenzwerte: 160,000 und 360,000), stieg nach der Operation bis zu 286,700 in $1 \mathrm{cmm}$ Blut (Grenzwerte : 210,000 und $370,000)$ an. Dabei wurde die Verminderung des Blutplättchens, die vor der Operation öfters vorkam, nach der Operation weit seltener 
und die Blutplättchenzahl näherte sich wie Normann und Foged u. a. bei anderweitigen Krankheiten nach chirurgischem Eingriff beobachtet, nach der Operation wieder der Norm. Dabei kam mitunter auch eine vorübergehende leichte Vermehrung des Blutplättchens direkt nach der Operation zur Beobachtung.

Bei den 10 beiderseitigen Nierentuberkulosen betrug das Blutplättchen durchschnittlich 220,000 in $1 \mathrm{cmm}$ Blut mit den Grenzwerten von 120,000 und 285,000 und die Verminderung, die dabei in der grössten Mehrzahl der Fälle eintrat, wurde oft der deutlichen Linksverschiebung der Neutrophilen oder mit der Verminderung der Erythrozytenzahl und des Hämoglobingehalts des Bluts begleitet.

Bei 9 Fällen der Nieren- und Uretersteinkrankheit verhielt sich die Blutplättchenzahl je nach der Schwere der anatomischen und funktionellen Schädigung des Nierenparenchyms durch mechanische Einwirkung des Steins sowie Harnstauung und Infektion verschieden. Im allgemeinen bemerkte ich aber dabei nur wenige Abweichungen von der Norm. Bei den Nierentumoren und Zystennieren war das Blutplättchen in allen Fällen vermindert, gleichzeitig von einer leichten Linksverschiebung der Neutrophilen begleitet. Bei Nephritis chronica haematurica wurden nur normale Werte der Blutplättchenzahl festgestellt. Diese war bei Pyelitis und Paranephritis acuta immer vermehrt als vermindert.

\section{Schluss.}

1. Ich bestimmte bei Gesunden und chirurgischen Kranken und zwar bei chirurgischen Nierenerkrankungen, also im ganzen bei 196 Personen, die Blutplättchenzahl nach der Methode von Lampert. Die Ergebnisse wurden mit dem gleichzeitig bestimmten Hämogramm sowie der Senkungsgeschwindigkeit der Erythrozyten verglichen.

2. Bei gesunden Erwachsenen betrug die Blutplättchenzahl durchschnittlich 300,000 in $1 \mathrm{cmm}$ Blut mit den Grenzwerten von 250,000 und 360,000 in $1 \mathrm{cmm}$ Blut. Bei vorgerücktem Alter wies die Blutplättchenzahl die unterste Grenze der Zahl bei gesunden Erwachsenen auf, während sich die Zahlen bei Säuglingen und Kindern durchschinittlich etwas höher als bei gesunden Erwachsenen verhielten.

3. Bei akuten eitrigen Infektionen wurde mehr oder weniger deutliche Thrombozytose unter Begleitung der Leukozytose und mässig starke Linksverschiebung der Neutrophilen beobachtet. Bei den schwersten Fällen trat dagegen Thrombopenie mit Leukopenie und der hochgradigen Linksverschiebung der Neutrophilen zu Tage. 
4. Bei Karzinomen und zwar bei Magenkarzinomen beobachtete ich im allgemeinen die Thrombopenie, die bei Metastasebildung noch deutlicher wurde. Die Magen- und Duodenalgeschwüre dagegen zeigten eine Neigung zur Thrombozytose. Bei Sarkomfällen liess sich eine mehr oder weniger starke Thrombozytose feststellen.

5. Bei Nierentuberkulosen und Nierengeschwülsten wurde immer eine deutliche Thrombopenie beobachtet, während das Blutplättchen bei Nephrolithiasis eine mehr erhöhte, aber innerhalb normaler Grenzen liegende Zahl zeigte. Bei einseitiger Nierentuberkulose war die Verminderung der Blutplättchenzahl im allgemeien und zwar bei geringer Parenchymzerstörung weniger ausgeprägt, kam dagegen bei stärkerer Zerstörung der erkrankten Niere noch deutlicher zu Tage. Bei beiderseitigen Nierentuberkulosen erwies sich die Thrombopenie auch mit der Alteration des Hämogramms und besonders des Leukozytenbildes als sehr hochgradig.

\section{Literatur.}

(1) Behr, C. H. ; Klin. Wochensehr., 1933, 1771-1774.

(2) Bokelmann, O. u. E. Nas s a ; Berlin. klin. Wochenschr,, 1918, 353-354.

(3) Dameshek, W.; Arch. Int. Med., 1932, 50, 579-589.

(4) Erdman n, D.; Ztschr. Urol., 1931, 25, 604-608.

(5) Foged, J.; Ugeskr. Laeg., 1930 II, 678-681. Ref.: Ztrlorg. ges. Chir., 1930, 51, 695 .

(6) Gundermann; Arch. klin. Chir., 1922, 121, 81-83.

(7) Helber, E.; Dtsch. Arch. klin. Med., 1904, 81, 316-327.

(8) Hueok, H.; Dtsch. Ztschr. Chir, 1925, 192, 322-329.

(9) Ka to, M. ; Rinsho Bỳorigaku Ketuekigaku Zassi, 1934, 3, 64-74.

(10) Kitasim a, K.; Kumamoto Igakkai Zassi, 1928, 4, 63-82.

(11) L a m pert; Verhdl. Dtsch. Gesell. inn. Med., 1931, 43, 92-93.

(12) Lam pert, H. ; Dtsch. med. Wochenschr., 1931, 2016-2017.

(13) Naege1i, 0.; Blutkrankheiten und Blutdiagnostik. 5. Aufl, Berlin 1931, 30-31 u. 180-189.

(14) Nisigori, $\mathrm{S}_{*}$; Rynsho, 1924-25, 2, 70-102.

(15) Norma n n, E,; Dtsch. Ztschr. Chir., 1928, 212, 166-178.

(16) Okamoto, T.; Rinsho Byorigaku Ketuekigaku Zassi, 1933, 2, 358-382.

(17) Perl, Ch.; Ztschr. klin. Med., 1932, 122, 253-256.

(18) Preiss, W.; Ztschr. exp. Med., 1932, 84, 810-823.

(19) Rosen baum, B. N.; Zbl. Chír.. 1924, 305-307.

(20) Schilling, V.; Das Blutbild und seine klinische Verwertung. 9. Aufl, Jena $1933,46-54$ u. 160.

(21) Tokuda, K. ; Tohoku Igaku Zassi, 1924-1925, 8, 512-568. 